\title{
KINK SOLUTIONS FOR THE NEWELL-WHITEHEAD-SEGEL EQUATION
}

\author{
M. A. Knyazev \\ Belarusian National Technical University, \\ 65, Independence Ave., Minsk, 220013, Belarus \\ e-mail: maknyazev@bntu.by
}

(Received February 04, 2019; in final form - May 26, 2019)

\begin{abstract}
Four kink (antikink) solutions for the Newell-Whitehead-Segel equation are constructed by the Hirota method for the special but general enough values of its parameters. The topological charges for these solutions are calculated. A possibility of the spontaneous symmetry breaking is pointed
\end{abstract} out.

Key words: kink, Newell-Whitehead-Segel equation, topological charge. DOI: https://doi.org/10.30970/jps.23.3001

PACS number(s): 02.30.Jr, 03.50.Kk

\section{INTRODUCTION}

The nonlinear Newell-Whitehead-Segel (NWS) equation was introduced in papers $[1,2]$ for studying the problems of fluid mechanics. It is well known that this equation is widely used in studying various mechanical, chemical and biological problems [3]. For $(1+1)$-dimensional case, it may be written in the form

$$
u_{t}=d u_{x x}+a u-b u^{q}
$$

where $u_{t}=\frac{\partial u}{\partial t}, u_{x x}=\frac{\partial^{2} u}{\partial x^{2}}, d, a$ and $b$ are real numbers, $q$ is a real, positive integer.

The have been many attempts to construct exact and approximative solutions to this equation analytically. In [4], an extension of amplitude NWS equation is considered. By the renormalization group method, it is shown that this equation is covariant under arbitrary rotations. A dispersive generalization of the NWS equation is used in [5] as an equation for almost onedimensional traveling-wave patterns. The homotopy perturbation method is applied to obtain the solution to the NWS equation in a closed form [6]. The Adomian decomposition method is used in [7] to find the approximate solution to the NWS equation for different coefficients. It is shown that this method leads to better results in comparison with the homotopy perturbation method. In [8], for two NWS equations this method was compared with the modified differential transform method. It was demonstrated that the Adomian transform method requires more computational work. The solutions of the NWS equations were constructed by He's variational iteration method in [9]. The perturbation iteration transform method is used in [10] for the solution of the NWS equation. The advantages of this method in comparison with some other methods are demonstrated. Not only was the construction of a new solution a subject of studying, but the properties of a class of the NWS equations are also considered with Lie and nonclassical symmetry in [11]. It allowed to establish the criteria of reducibility of the NWS equations with variable coefficients to their constant coefficient counterparts.
A specific feature of Eq.(1) is that it is not possible to construct its localized traveling-wave solution for the arbitrary values of its parameters [5]. In this paper, the four new kink and/or antikink solutions of the NWS equation are obtained for the case when $d, a, b$ are real positive numbers and $q=3$. This is a general enough case (only $q$ has a special value but, as it is demonstrated below, this is a natural value for the method applied). To construct these solutions, the direct Hirota method is used $[12,13]$. In Sec. II, this method is described in brief. In Sec. III, the new solutions are constructed in an explicit form. In Sec. IV, some concluding remarks are presented.

\section{METHOD OF SOLUTION}

Let us consider the NWS equation Eq.(1)when $d, a, b$ are real positive numbers. Let us introduce a new function by the Cole-Hopf transformation

$$
u(x, t)=\sigma \frac{F_{x}}{F},
$$

where $F=F(x, t)$ is a new unknown function, $\sigma$ is a constant parameter to be determined below, $F_{x}=\frac{\partial F}{\partial x}$. By substituting Eq.(2) into Eq.(1), the latter equation may be written in the form

$$
\begin{aligned}
\sigma \frac{F_{x t}}{F} & -\sigma \frac{F_{x} F_{t}}{F^{2}}-\sigma d \frac{F_{x x x}}{F}+3 \sigma d \frac{F_{x} F_{x x}}{F^{2}} \\
& -2 \sigma d \frac{F_{x}^{3}}{F^{3}}-\sigma a \frac{F_{x}}{F}+b \sigma^{q} \frac{F_{x}^{q}}{F^{q}}=0
\end{aligned}
$$

As the purpose of this paper is to construct a special solution for Eq.(1), then it is possible to introduce a special condition for Eq.(3). This condition may be written as

$$
b \sigma^{q} \frac{F_{x}^{q}}{F^{q}}-2 \sigma d \frac{F_{x}^{3}}{F^{3}}=0 .
$$

It is clear that for Eq.(4) the value $q=3$ is a natural. For this value of $q$ from Eq.(4) the following two values 
of parameter $\sigma$ may be obtained

$$
\sigma= \pm \sqrt{\frac{2 d}{b}}
$$

Now Eq. (3) reduces to the bilinear equation of the form

$$
F_{x t} F-F_{x} F_{t}-d F_{x x x} F+3 d F_{x} F_{x x}-a F_{x} F=0 .
$$

The next step is a usual one for the Hirota method. Let us represent $F(x, t)$ as a formal series

$$
F=1+\varepsilon f_{1}+\varepsilon^{2} f_{2}+\varepsilon^{3} f_{3}+\ldots
$$

where $f_{i}, i=1,2,3, \ldots$ are new unknown functions, $\varepsilon$ not necessarily a small constant. By substituting Eq. (7) into Eq. (6) and equating the coefficients to zero for each power of $\varepsilon$, an infinite system of linear partial differential equations for the functions $f_{i}$ will be obtained. The first three equations of this system may be written as

$$
\begin{gathered}
f_{1, x t}-d f_{1, x x x}-a f_{1, x}=0, \\
f_{2, x t}-d f_{2, x x x}-a f_{2, x}=-3 d f_{1, x} f_{1, x x}+f_{1, x} f_{1, t}, \\
f_{3, x t}-d f_{3, x x x}-a f_{3, x}=f_{1, x} f_{2, t}+f_{1, t} f_{2, x}-3 d f_{1, x} f_{2, x x} \\
-3 d f_{1, x x} f_{2, x}-f_{1} f_{2, x t}+d f_{1} f_{2, x x x}+a f_{1} f_{2, x} .
\end{gathered}
$$

For every $i$ the function $f_{i}$ is determined by the previous functions and their derivatives only. Only the first equation of this system is homogeneous, the others are not. If the Hirota method may be applied to solve some nonlinear equation, the problem of an exact truncation of the series (7) is not essential.

\section{ONE-KINK SOLUTIONS}

To construct a one-kink solution, Eq. (8) and Eq. (9) are needed. Let us represent function $f_{1}$ in the form

$$
f_{1}(x, t)=\exp \left(k_{1} x-\omega_{1} t+\eta_{1}^{0}\right)
$$

where $k_{1}, \omega_{1}$ and $\eta_{1}^{0}$ are the parameters of the solution to be determined. $\eta_{1}^{0}$ is an initial phase shift, and without the loss of generality we may equal it to zero. Now the one-kink solution may be written as

$$
u(x, t)=\sigma \frac{f_{1, x}}{1+f_{1}} .
$$

By substituting Eq. (11) into Eq. (8), we can obtain the following relation between $k_{1}$ and $\omega_{1}$ :

$$
\omega_{1}=-d k_{1}^{2}-a
$$

This is a dispersion relation. The second linearly independent equation for $k_{1}$ and $\omega_{1}$ may be obtained by substituting Eq. (11) into the right-hand side of Eq. (9) and equating it to zero. This equation is of the form

$$
\omega_{1}=-3 d k_{1}^{2} \text {. }
$$

The exact values of parameters $k_{1}$ and $\omega_{1}$ may be found from these two last equations:

$$
k_{1}= \pm \sqrt{\frac{a}{2 d}} .
$$

For $k_{1}$ of both signs

$$
\omega_{1}=-\frac{3 a}{2} .
$$

Now the four one-kink(antikink) solutions for Eq. (1) may be written as:

$$
\begin{aligned}
& \text { 1) } \sigma=\sqrt{\frac{2 d}{b}}, k_{1}=\sqrt{\frac{a}{2 d}}, \omega_{1}=-\frac{3 a}{2} \\
& u(x, t)=\frac{1}{2} \sqrt{\frac{a}{b}}\left\{1+\tanh \left[\frac{1}{2}\left(\sqrt{\frac{a}{2 d}} x+\frac{3 a}{2} t\right)\right]\right\}, \\
& \text { 2) } \sigma=\sqrt{\frac{2 d}{b}}, k_{1}=-\sqrt{\frac{a}{2 d}}, \omega_{1}=-\frac{3 a}{2} \\
& u(x, t)=-\frac{1}{2} \sqrt{\frac{a}{b}}\left\{1+\tanh \left[\frac{1}{2}\left(-\sqrt{\frac{a}{2 d}} x+\frac{3 a}{2} t\right)\right]\right\}, \\
& \text { 3) } \sigma=-\sqrt{\frac{2 d}{b}}, k_{1}=\sqrt{\frac{a}{2 d}}, \omega_{1}=-\frac{3 a}{2} \\
& u(x, t)=-\frac{1}{2} \sqrt{\frac{a}{b}}\left\{1+\tanh \left[\frac{1}{2}\left(\sqrt{\frac{a}{2 d}} x+\frac{3 a}{2} t\right)\right]\right\}, \\
& \text { 4) } \sigma=-\sqrt{\frac{2 d}{b}}, k_{1}=-\sqrt{\frac{a}{2 d}}, \omega_{1}=-\frac{3 a}{2} \\
& u(x, t)=\frac{1}{2} \sqrt{\frac{a}{b}}\left\{1+\tanh \left[\frac{1}{2}\left(-\sqrt{\frac{a}{2 d}} x+\frac{3 a}{2} t\right)\right]\right\} .
\end{aligned}
$$

It is easy to verify by using direct substitution that Eqs. (17)-(20) are the solutions of Eq. (1). If one of the solutions is constructed, the others may be obtained due to the symmetries of the Eq. (1): $x \rightarrow-x$ and $u \rightarrow-u$.

\section{CONCLUSION}

The four solutions of Eq. (1) are constructed. Eq. (17) describes an usual kink solution and Eq. (20) describes the corresponding antikink solution. As for the other two solutions, by analogy with the approach used in the soliton theory, they may be called a dark kink solution [Eq. (19)] and a dark antikink solution [Eq. (18)]. Eq. (1) contains the damping term, nevertheless the form and velocity of propagation for every solution (17)-(20) are conserved. It means that all these solutions are stable [14]. If the conditions under which these solutions are obtained vary, the localized traveling-wave solution cannot always be constructed but the oscillating solutions appear.

All the solutions (17)-(20) are not static. If we consider a static case $\left(u_{t}=0\right)$, then Eq. (1) takes the form of the static equation of $\phi^{4}$-theory:

$$
u_{x x}+\frac{a}{d} u-\frac{b}{d} u^{3}=0 .
$$


The potential of such a model equals to zero at two minima: $u= \pm \sqrt{\frac{a}{b}}$. Eq. (21) has two solutions:

$$
u(x)= \pm \sqrt{\frac{a}{b}} \tanh \left[\sqrt{\frac{a}{2 d}}\left(x-x_{0}\right)\right] .
$$

In this equation, "+" corresponds to the kink solution, "" corresponds to the antikink solution. $x_{0}$ is the position of the solution at an initial moment of time. Hence, the analysis of topological charges $Q$ for Eq. (1) in a static case is similar to such an analysis for $\phi^{4}$-theory [15].

If we consider a non-static case $\left(u_{t} \neq 0\right)$, then Eq. (1) describes a non-conservative system (it contains a dissipative term). Nevertheless, the topological charges may be determined as well. For us it is important that the solutions with the same topological charge belong to the same topological sector. Let us determine the topological charge according to [15]:

$$
Q=2 \sqrt{\frac{b}{a}}[u(x=\infty)-u(x=-\infty)] .
$$

As a result, one obtains that kink and dark antikink possess the topological charge $Q=2$ and dark kink and antikink possess the topological charge $Q=-2$. This result indicates that the potential of NWS-equation has two minima. It also indicates the possibility of the spontaneous symmetry breaking for the system described by NWS-equation.
In the paper the solutions in a form of travelling waves are constructed. It is possible to reduce Eq. (1) to an ordinary differential equation. This may simplify the calculations from the very beginning. Nevertheless, our approach is more prospective for the purpose of constructing the solutions corresponding to the coupled states. An attempt to construct solutions corresponding to a coupled state of two kinks (two antikinks or kink-antikink)by the Hirota method faces some problems. For this purpose a function $F=1+f_{1}+f_{2}$ is needed. Hence, function $f_{2}$ should be determined. It has the form

$$
f_{2}=\exp \left(\eta_{1}+\eta_{2}+A_{12}\right)
$$

where $A_{12}=\frac{\left(k_{1}-k_{2}\right)^{2}}{\left(k_{1}+k_{2}\right)^{2}}, \eta_{i}=k_{i} x-\omega_{i} t+\eta_{i}^{0}, i=1,2$. For the problem under consideration, parameters $k_{1}$ and $k_{2}$ have exact and fixed values. They equal each other up to a sign. That is why term $A_{12}$ is equal to 0 or $\infty$. When $A_{12}=0$ we have not a coupled state but a usual sum of two single objects (for example, like kinks). When $A_{12}=\infty$, the contribution of $f_{2}$ to the coupled state cannot be calculated. By using a generalization of the Hirota method [16], it is possible to construct the solution similar to coupled state of kinks or antikinks.

Acknowledgment. I would like to acknowledge the anonymous referee for the useful remarks which improved the paper.
[1] A. Newell, J. Whitehead, J. Fluid Mechan. 38, 279 (1969); https://doi.org/10.1017/S0022112069000176.

[2] L. A. Segel, J. Fluid Mechan. 38, 203 (1969); https: //doi.org/10.1017/S0022112069000127.

[3] J. D. Murray: Mathematical Biology I: An Introduction (Spinger, New York, 2002).

[4] R. Graham, Phys. Rev. Lett. 76, 2185 (1996); https://doi.org/10.1103/PhysRevLett. 76.2185; erratum: Phys. Rev. Lett. 80, 3888 (1998); https://doi.org/10.1103/PhysRevLett.80.3888.

[5] B. A. Malomed, preprint arXiv:patt-sol/9605001 (1996).

[6] S. S. Nourazar, M. Soori, A. Nazari-Golshan, Aust. J. Basic Appl. Sci. 5, 1400 (2011).

[7] A. A. Aswhad, A. F. Jaddoa, Al-Mustansiriyah J. Sci. 25, 45 (2014); https://doi.org/10.23851/mjs.v.25i1.

[8] A. Saravanan, N. Magesh, J. Egypt Math. Society 21, 259 (2013); https://doi.org/10.1016/j.joems. 2013. 03.004.

\section{РОЗВ'ЯЗКИ КІНКОВОГО ТИПУ ДЛЯ РІВНЯННЯ НЬЮЕЛЛА-ВАЙТГЕДА-СЕГЕЛА}

\section{М. Князєв}

Білорусъкий національний технічний університет, просп. Незалежності, 65, Мінсък, 220013, Білорусь

Для рівняння Ньюелла-Вайтгеда-Сегела за методом Гіроти побудовано чотири розв'язки у вигляді кінків (антікінків) для спеціальних, але досить загальних значень параметрів. Обчислено топологічні заряди, що відповідають цим розв'язкам. Показано можливість спонтанного порушення симетрії в системі, яку описує розглянуте рівняння.
[9] A. Prakash, M. Kumar, J. Appl. Anal. Comput. 6, 738 (2016); https://doi.org/10.11948/2026048.

[10] G. O. Akinlabi, S. O. Edek, J. Math. Stat. 13, 24 (2017); https://doi.org/10.3844/jmssp.201.24.29.

11] O. Vaneeva, V. Boyko, A. Zhalji, C. Sophocleous, J. Math. Anal. Appl. 474, 264 (2019); https://doi.org/ j.jmaa. 2019.01.044.

M. J. Ablowitz, H. Segur, Solitons and Inverse Scattering Transform (SIAM, Philadelphia, 1981).

3] E. Infeld, G. Rowlands: Nonlinear Waves, Solitons and Chaos (Cambridge University Press, 2000). 435 (1986); https://doi.org/10.1016/0375-9601(86) 90688-2. Publ. Co., 1982.

M. A. Knyazev, Kinks in the Scalar Model with Damping (Tekhnologia, Minsk, 2003) [in Russian].

\footnotetext{
суе розглянуге рівняння.
} 